\title{
Review of staging and diagnosis of malignant pleural mesothelioma
}

\author{
Sean Jordan ${ }^{1}$, Lindsay Nitsche $^{2} \wedge$, Sai Yendamuri ${ }^{2}$ \\ ${ }^{1}$ University of Tennessee Medical Center, Knoxville, TN, USA; ${ }^{2}$ Roswell Park Comprehensive Cancer Center, Buffalo, NY, USA \\ Contributions: (I) Conception and design: All authors; (II) Administrative support: L Nitsche, S Jordan; (III) Provision of study materials or patients: \\ All authors; (IV) Collection and assembly of data: All authors; (V) Data analysis and interpretation: S Yendamuri, S Jordan; (VI) Manuscript writing: \\ All authors; (VII) Final approval of manuscript: All authors. \\ Correspondence to: Sai Yendamuri, MD, FACS. Professor and Chair of Thoracic Surgery, Roswell Park Comprehensive Cancer Center, Buffalo, NY, \\ USA. Email: sai.yendamuri@roswellpark.org.
}

\begin{abstract}
Despite the aggressive nature of malignant pleural mesothelioma (MPM) its diagnosis and accurate staging remains elusive. MPM is highly associated with asbestos exposure, but the long latency period makes exposure tracking a challenge. The disease often presents surreptitiously leading to late diagnosis and therefore poor prognosis. Patients often present with symptoms such as dyspnea, chest pain, fatigue, and cough that are suggestive of many thoracic ailments such as lung cancer, pneumothorax, and pneumonia. Consequently, these patients often receive a computerized tomography (CT) scan as the first step towards diagnosis. If the results of the CT scan are suggestive of mesothelioma, such as if diffuse pleural thickening or a rind is seen, a biopsy is the next intervention sought. A thoracoscopic biopsy is often preferred over a core needle biopsy and is paramount for diagnosis and subsequent treatment plan optimization. Positron emission tomography (PET) scans have been useful in determining the spread of the disease while magnetic resonance imaging (MRI) has been a tool in determining respectability. In an effort to optimize care for these patients, staging guidelines have evolved and now align with those utilized with solid tumors such as sarcoma. The current classification system is the eighth edition of the tumor, node, and metastasis (TNM) system published by the International Mesothelioma Interest Group (IMIG) for MPM. This edition incorporates changes in the $\mathrm{T}$ and $\mathrm{N}$ classifications and differs from previous guidelines that focused on surgical management of the disease. In an effort to improve clinical decision making and prognostication novel staging approaches, including volumetric staging, have been proposed. Diagnosis and treatment of MPM is further complicated by the difference in behavior and tumor marker expression of the epithelioid subtype and the sarcomatoid subtypes. Utilizing computerized databases, literature pertaining to the diagnosis and staging of MPM was analyzed. We focused on the utility of current imaging strategies, staging systems, and pathologic analysis. MPM remains a challenge to diagnose and treat. The increased integration of advanced imaging modalities will likely aid in diagnosis of MPM. Advancements in diagnosis will predictably go hand-in-hand with more precise staging systems that will overall improve prognostication and survival.
\end{abstract}

Keywords: Mesothelioma; cancer staging; video-assisted thoracoscopic surgery; cancer imaging

Received: 26 July 2021; Accepted: 20 March 2022; Published: 30 September 2022.

doi: 10.21037/asj-21-71

View this article at: https://dx.doi.org/10.21037/asj-21-71

$\wedge$ ORCID: 0000-0001-5733-3103. 


\section{Introduction}

Malignant pleural mesothelioma (MPM) is an aggressive malignancy that remains a challenge to diagnose. Symptoms for patients are usually insidious and frequently mimic other benign and malignant chest diseases. A prudent example is early stage mesothelioma masquerading with symptoms suggestive of primary pneumothorax which may lead to tardy diagnosis. Suspicion must be high for any clinical provider who encounters a patient with respiratory or chest complaints such as dyspnea, pain, unexplained pneumothorax and a history of asbestos exposure. Computerized tomography (CT) is an important imaging modality for evaluating a patient with suspected MPM, but tissue diagnosis is critical, and in some countries is required in order to get compensation for occupational hazard exposure. In addition, tissue diagnosis provides important information for prognostication and treatment decisions. Staging for MPM has undergone many changes over the last few decades, evolving from systems primarily focused on surgical management of the disease to a tumor, node, and metastasis (TNM) system more consistent with other solid tumors.

\section{Staging systems}

Older mesothelioma staging systems include the Brigham and the Butchart systems. The Brigham staging system was proposed by Sugarbaker and colleagues in the late 1990s in response to perceived problems with the International Mesothelioma Interest Group (IMIG) system at the time and was based on tumor, resectability, and nodal status and was derived from patients who underwent extrapleural pneumonectomies (EPP) at a single institution (1-3). It was not widely accepted due to its reliance on surgical margins in patients undergoing EPP and because it differed from the typical TNM staging guidelines (4). The Butchart system was developed in the 1970s and was more simplistic but nevertheless insightful and defined four stages: (I) tumor only present on the ipsilateral pleura, lung, and pericardium on the ipsilateral side, (II) tumors that invade the mediastinum or mediastinal structures, (III) tumor that traverse the diaphragm and enter the peritoneum or involve extrathoracic lymph nodes, or (IV) distant hematogenous metastases (4). Presently, the IMIG system is the most commonly used.

\section{Clinical TNM staging}

The first widely accepted TNM classification for MPM was published by the IMIG in 1994 as a result of a consensus meeting of the Seventh World Conference of the International Association for the Study of Lung Cancer (IASLC) (5). It was the first MPM staging system that utilized the outcomes of multiple retrospective series. This system was ultimately adopted and revised by the American Joint Commission on Cancer (AJCC) and the Union for International Cancer Control (UICC) (6,7). As of 2021, the TNM system published by the IMIG for MPM is currently on its eighth edition.

Compared to the seventh edition, the $\mathrm{T}$ stage in the eighth edition has been simplified based on more recent data (Table 1) (6). In the seventh edition, T1a was defined as tumor involving the ipsilateral parietal pleura without involvement of the visceral pleura and $\mathrm{T} 1 \mathrm{~b}$ was tumor involving the parietal pleura on the ipsilateral side with focal involvement of the visceral pleura. In the eighth edition, this has been condensed and simplified to just $\mathrm{T} 1$, which is defined as tumor involving the ipsilateral parietal pleura with or without involving of the visceral pleura. The other stages remain unchanged. T2 refers to tumor that involves the ipsilateral pleural surfaces (parietal, mediastinal, diaphragmatic, or visceral) and one or more of the following: confluent visceral pleural tumor, involvement of the diaphragm muscle, and/or invasion of the lung parenchyma. T3 refers to involvement of all the ipsilateral pleural surfaces with at least one of the following: invasion of the endothoracic fascia, extension into mediastinal fat, solitary completely resectable focus invading soft tissues of the chest wall, and/or non-transmural involvement of the pericardium. T4 refers to involvement of all the ipsilateral pleural surfaces with at least one of the following: diffuse or multifocal invasion of the chest wall's soft tissue, involvement of ribs, invasion through the diaphragm into the peritoneum, invasion of any mediastinal organ, direct extension to the pleura on the contralateral side, invasive of brachial plexus, the spine, or transmural invasion of the pericardium. The transmural involvement may or may not have a concomitant pericardial effusion. Invasion into the myocardium would also classify the tumor as T4. When updating this classification system, the IASLC did review data that showed there was a significant survival benefit when no pleural thickness was greater than $5.1 \mathrm{~mm}$ versus any pleural thickness greater than $5.1 \mathrm{~mm}$ (median survival 24.2 vs. 17.7 months, $\mathrm{P}=0.0014$ ), however, it was not included due to need for further analysis and the somewhat 
Table 1 Comparison of T characterizes between the eight and seventh editions of the IASLC TNM guidelines

\begin{tabular}{|c|c|c|}
\hline T Category & Seventh edition classification & Eighth edition classification \\
\hline TO & No evidence of primary tumor & No evidence of primary tumor \\
\hline T1a & $\begin{array}{l}\text { Tumor involves the parietal pleura on the ipsilateral side } \\
\text { (which includes that of the mediastinum and diaphragm) } \\
\text { with no involvement of visceral pleura }\end{array}$ & $\begin{array}{l}\text { Tumor involves the parietal pleura on the ipsilateral side } \\
\text { (which includes that of the mediastinum and diaphragm) } \\
\text { with no involvement of visceral pleura }\end{array}$ \\
\hline T2 & $\begin{array}{l}\text { Tumor involves parietal, mediastinal, diaphragmatic and } \\
\text { visceral pleural surfaces on the ipsilateral side and is } \\
\text { also can be characterized by any of the listed qualities: } \\
\text { - Tumor that impacts the entirety of the visceral pleura } \\
\text { (including the fissures) } \\
\text { - Tumor that effects the diaphragm } \\
\text { - Tumor that has invaded the parenchyma of the lung }\end{array}$ & $\begin{array}{l}\text { Tumor involves parietal, mediastinal, diaphragmatic and } \\
\text { visceral pleural surfaces on the ipsilateral side and is also } \\
\text { can be characterized by any of the listed qualities: } \\
\text { - Tumor that impacts the entirety of the visceral pleura } \\
\text { (including the fissures) } \\
\text { - Tumor that effects the diaphragm } \\
\text { - Tumor that has invaded the parenchyma of the lung }\end{array}$ \\
\hline T3 & $\begin{array}{l}\text { Tumor involves parietal, mediastinal, diaphragmatic and } \\
\text { visceral pleural surfaces on the ipsilateral side and is } \\
\text { also can be characterized by any of the listed qualities: } \\
\text { - Tumor invades that pericardial fascia, but is not } \\
\text { transmural } \\
\text { - Tumor invades the chest wall in a single location that } \\
\text { is amenable to resection } \\
\text { - Tumor invades into the mediastinal fat } \\
\text { - Tumors invades into the endothoracic fascia }\end{array}$ & $\begin{array}{l}\text { Tumor involves parietal, mediastinal, diaphragmatic and } \\
\text { visceral pleural surfaces on the ipsilateral side and is also } \\
\text { can be characterized by any of the listed qualities: } \\
\text { - Tumor invades that pericardial fascia, but is not } \\
\text { transmural } \\
\text { - Tumor invades the chest wall in a single location that is } \\
\text { amenable to resection } \\
\text { - Tumor invades into the mediastinal fat } \\
\text { - Tumors invades into the endothoracic fascia }\end{array}$ \\
\hline $\mathrm{T} 4$ & $\begin{array}{l}\text { Tumor involves parietal, mediastinal, diaphragmatic and } \\
\text { visceral pleural surfaces on the ipsilateral side and is } \\
\text { also can be characterized by any of the listed qualities: } \\
\text { - Tumor invades the chest wall diffusely or in a way not } \\
\text { amenable to resection } \\
\text { - Tumor extends to contralateral pleura } \\
\text { - Tumor involves a rib } \\
\text { - Tumor progresses through the diaphragm } \\
\text { - Tumor invades any mediastinal organ, spine, or the } \\
\text { brachial plexus } \\
\text { - Tumor involves the entire thickness of the pericardium } \\
\text { +/- effusion } \\
\text { - Tumor with myocardial involvement }\end{array}$ & $\begin{array}{l}\text { Tumor involves parietal, mediastinal, diaphragmatic and } \\
\text { visceral pleural surfaces on the ipsilateral side and is also } \\
\text { can be characterized by any of the listed qualities: } \\
\text { - Tumor invades the chest wall diffusely or in a way not } \\
\text { amenable to resection } \\
\text { - Tumor extends to contralateral pleura } \\
\text { - Tumor involves a rib } \\
\text { - Tumor progresses through the diaphragm } \\
\text { - Tumor invades any mediastinal organ, spine, or the } \\
\text { brachial plexus } \\
\text { - Tumor involves the entire thickness of the pericardium } \\
+ \text { - effusion } \\
\text { - Tumor with myocardial involvement }\end{array}$ \\
\hline
\end{tabular}

IASLC, International Association for the Study of Lung Cancer; TNM, tumor, node, and metastasis.

subjective nature of the measurements on the collected data (8).

Just as with lung cancer, the $\mathrm{N}$ staging system is important for prognostication for survival in patients with MPM. In the seventh edition, the classification system was N0-3. In the eighth edition, N3 was consolidated with N2 (Table 2). N1 correlates with nodal metastases in the ipsilateral bronchopulmonary, hilar, or mediastinal lymph nodes. N2 now correlates with metastases in the contralateral bronchopulmonary, hilar, or mediastinal lymph nodes or any supraclavicular lymph nodes. N3 is no longer used. For the $M$ system, M1 continues to refer to any distant metastases (Table 3). Major sites for metastasis include lung, liver, adrenal gland, kidneys, spleen, thyroid, and brain (9).

\section{Patbologic staging}


Table 2 Comparison of $\mathrm{N}$ characterizes between the eight and seventh editions of the IASLC TNM guidelines

\begin{tabular}{lll}
\hline N Category & Seventh edition classification & Eighth edition classification \\
\hline Nx & Cannot assess regional lymph nodes & Cannot assess regional lymph nodes \\
N0 & No metastasis to regional lymph nodes & No metastasis to regional lymph nodes \\
& $\begin{array}{l}\text { The hilar lymph nodes or the bronchopulmonary nodes on } \\
\text { the ipsilateral side show evidence of metastasis }\end{array}$ & $\begin{array}{l}\text { The hilar, mediastinal, or the bronchopulmonary lymph } \\
\text { nodes on the ipsilateral side. Show evidence of metastasis } \\
\text { (the intercostal, pericardial fat pad, internal mammary, peri- } \\
\text { diaphragmatic, lymph nodes are also included here) }\end{array}$ \\
& & The mediastinal lymph nodes, subcarinal lymph nodes, \\
N2 & $\begin{array}{l}\text { internal mammary nodes on the ipsilateral side, or } \\
\text { the peridiaphragmatic lymph nodes show evidence of }\end{array}$ & $\begin{array}{l}\text { or mediastinal lymph nodes or ipsilateral or contralateral } \\
\text { supraclavicular lymph nodes }\end{array}$ \\
& $\begin{array}{l}\text { metastasis } \\
\text { The scalene or supraclavicular lymph nodes on the }\end{array}$ & N/A \\
& ipsilateral side or, the hilar lymph nodes, contralateral \\
internal mammary, contralateral mediastinal show evidence & \\
& of metastasis &
\end{tabular}

IASLC, International Association for the Study of Lung Cancer; TNM, tumor, node, and metastasis.

Table 3 Comparison of $M$ characterizes between the eight and seventh editions of the IASLC TNM guidelines

\begin{tabular}{lll}
\hline M Category & Seventh edition classification & Eighth edition classification \\
\hline Mx & Distant metastasis existence cannot be assessed & Distant metastasis existence cannot be assessed \\
M0 & Distant metastases not evident & Distant metastases not evident \\
M1 & Distant metastases evident & Distant metastases evident \\
\hline
\end{tabular}

IASLC, International Association for the Study of Lung Cancer; TNM, tumor, node, and metastasis.

Thoracentesis and analysis of body fluid cytology is frequently done for pleural effusions of unknown origin. In MPM, diagnosis can be challenging as cytologic features tend to be bland and the tissue invasion that is usually the reliable morphologic criteria for MPM cannot be assessed in a cytology preparation (10). Cytologic diagnosis is also limited to epithelioid subtypes as sarcomatoid subtypes do not shed fluid into the pleural effusions. The American Society of Clinical Oncology practice guidelines published in 2018 by Kindler et al. recommend that cytologic evaluation can be an early screening test for mesothelioma but argued against its use as a diagnostic test due to lack of sensitivity (11). Loss of BAP1 and/or deletion of p16 seen in mesothelioma, but not reactive mesothelial cells, could be a useful adjunct for cytologic diagnosis (12).

Core needle biopsy is an option for diagnosis. Imageguided core needle biopsy using ultrasound or CT can have a sensitivity of $77-88 \%$ with a needle track seeding rate of $0-4 \%(13-15)$. In patients with easily accessible pleural masses or who are higher risk for thoracoscopic biopsy, image-guided core needle biopsy is an appropriate first step. Fine needle biopsy has a sensitivity of only $30 \%$ and is not recommended (16).

Thoracoscopic biopsy remains the gold standard for obtaining tissue diagnosis for mesothelioma. It offers advantages over core needle biopsy such as the ability to gather more tissue for evaluation, ability to assess resectability by analyzing invasion and disease extent, and it affords the ability to perform talc pleurodesis to mitigate recurrent pleural effusion (17). VATS pleural biopsy has a sensitivity of $95 \%$, specificity of $100 \%$, and NPV of $94 \%$ (18). This is similar to medical pleuroscopy/ thoracoscopy, although data directly comparing the two is lacking and there likely isn't a significant difference. Thoracoscopy under purely local anesthetic is a feasible option and pooled evaluation from 1,369 patients from 22 studies by Rahman et al. showed a sensitivity of $92 \%$ for diagnosing malignant pleural disease (19). A randomized controlled trial involving 58 patients by Haridas et al. 
showed that medical thoracoscopy had a diagnostic yield of $86 \%$ with a complication rate of $10 \%$ compared to $62 \%$ and $17 \%$ respectively for closed Abram needle biopsy (20). Whenever VATS biopsy is performed, incision planning should be performed with possible surgical treatment in mind, and it is generally recommended that the VATS incisions should be performed in line with future thoracotomy sites (21). Tumor spread at resected previous incisions has been described as a negative prognostic indicator for long term survival (22). Occasionally, due to an obliterated pleural space from advanced disease, a musclespearing incision within an intercostal space must be used for an open pleural biopsy.

Determining nodal status prior to operative intervention remains a challenge in MPM. Rice et al. compared cervical mediastinoscopy with endobronchial ultrasound (EBUS) with or without esophageal endoscopic ultrasound (EUS) (23). In examining 85 patients who underwent mediastinoscopy and/or EBUS, they found that mediastinoscopy had a sensitivity of $28 \%$ and negative predictive value (NPV) of $49 \%$, whereas EBUS had a sensitivity of $59 \%$ and NPV of $57 \%$. Eleven patients had EUS due to clinical suspicion on CT or positron emission tomography (PET), of whom infradiaphragmatic nodal metastases were identified in 5. Czarnecka-Kujawa et al. examined 48 patients who underwent EBUS only, have found a sensitivity of $16.7 \%$, specificity of $100 \%$, positive predictive value (PPV), and NPV of $70.6 \%$ (24). In their group, preoperative mediastinal lymph node staging prevented surgical resection in $18.8 \%$ by detection of $\mathrm{N} 2$ or N3 disease under the $7^{\text {th }}$ edition IMIG staging system. As with lung cancer, tissue-based mediastinal lymph node evaluation prior to surgical resection should be strongly considered based on clinical suspicion.

Blood based biomarkers have only a limited utility in the diagnosis of MPM presently. Mesothelin is a cell-adhesion glycoprotein typically over-expressed in MPM (25). Mesothelin levels in the serum are elevated in patients compared to control patients with a history of asbestos exposure. Epithelioid tumors are more likely to express mesothelin compared to tumors with sarcomatoid sub-types, which rarely express it. In a meta-analysis by Hollevoet et al. of 4,491 patients of whom 1,026 had MPM, the sensitivity of serum mesothelin was $32 \%$ with a $95 \%$ specificity (26). In another meta-analysis by Cui et al., pleural fluid mesothelin had a sensitivity of $61 \%$ and specificity of $87 \%$ (27). Serum mesothelin is not used frequently but when elevated should prompt further workup, although a negative test should be regarded skeptically. There has been some interest in using serum mesothelin as a screening tool in asbestos-exposed populations, but prospective studies have failed to demonstrate value for this $(26,28,29)$. Soluble mesothelin-related protein (SMRP) is another plasma biomarker that is Food and Drug Administration (FDA) approved for diagnosis of MPM, and other biomarkers such as megakaryocyte potentiating factor (MPF), osteopontin, fibulin-3, calretinin, and High-Motility Group Box 1 (HMGB1) are under investigation (30,31).

\section{Volumetric staging}

Though clinical TNM (cTNM) staging is the current modality by which we assess MPM patients it has been shown to be unreliable and suboptimal. In a study of 472 patients cTNM and pathological TNM (pTNM) were concordant in $36.2 \%$ with $44.2 \%$ and $19.4 \%$ of patients being overstaged or understaged respectively (32). A possible alternative suggests the utilization of volumetric analysis of the patient's CT scan, volumetric computed tomography (VolCT). VolCT involves the creation of a 3D model of tumor burden derived from data acquired from CT scans (32). VolCT was shown to have better correlation between different radiologists than cTNM (33). VolCT was also shown to have improved prognostic performance when compared to cTNM (P=0.001) (32).

\section{Image modalities for diagnosis and staging}

With the staging parameters in mind, the importance of imaging in the non-invasive staging of MPM becomes paramount. As with most solid malignancies, imaging provides crucial information for determining the extent of disease.

$\mathrm{CT}$ is the most commonly and widely using imaging modality for the diagnosis and staging of mesothelioma. Unilateral pleural effusion, pleural thickening, and invasion of structures such as the chest wall or mediastinum are the usual findings. Limitations of CT imaging include difficulty estimating chest wall and mediastinal invasion and involvement of the peritoneal cavity. An additional limitation is that CT imaging cannot often differentiate between pleural effusion secondary to benign or malignant causes. Involvement of lymph nodes is also difficult to estimate with CT scan alone.

In 1990, Kawashima et al. wrote a pictorial essay that examined the pretreatment $\mathrm{CT}$ findings of 50 patients 
(mostly men) with MPM (34). The most common CT images that group found included in order of decreasing frequency: pleural thickening (92\%), thickening of the interlobar fissure (86\%), unilateral pleural effusion (74\%), thoracic lymph nodes $1 \mathrm{~cm}$ or larger in maximum diameter $(58 \%)$ contraction of the involved hemithorax (42\%), pleural calcification (which is not a manifestation of mesothelioma, but one of benign asbestos exposure) (20\%), chest wall invasion (18\%), contralateral mediastinal shift $(14 \%)$, direct retroperitoneal invasion (8\%), pericardial effusion $(6 \%)$, and extension of tumor to the contralateral hemithorax (4\%). Sahin et al. in 1993 examined 82 patients in Turkey with MPM and history of exposure to asbestos or zeolite and reported similar CT findings and frequency including pleural thickening, nodules, or masses (100\%), mediastinal pleural involvement (93\%), involvement of intralobar fissures $(76 \%)$, volume contraction of the involved hemithorax (73\%), pleural effusions (73\%), pleural calcifications $(62 \%)$, contralateral hyaline plaques (26\%), rib erosion (19\%), and pneumothorax (4\%) (35).

Whenever the above findings are found without an explanation, suspicion must be raised for MPM, even with negative cytologic findings (36). It is difficult to differentiate MPM from other malignancies or even benign disease based only on the above findings, however. There is the risk for false positive cases such as biopsy proven disease in a patient who is very unlikely to have mesothelioma such as a young woman with a spontaneous pneumothorax. Results should be analyzed critically with patient specific information in mind. Leung et al. analyzed 74 patients (39 malignant, 35 benign) and found that patients with malignant pleural disease were more likely to have circumferential pleural thickening or rind (sensitivity $41 \%$, specificity $100 \%$ ), nodular pleural thickening (sensitivity $51 \%$, specificity $94 \%$ ), parietal pleural thickening of more than $1 \mathrm{~cm}$ (sensitivity $36 \%$, specificity $94 \%$ ), and mediastinal pleural involvement (sensitivity $56 \%$, specificity $88 \%$ ) (37). That group found all of those features were significantly more common in patients with malignant rather than benign pleural thickening. Certain infectious processes such as actinomycosis, tuberculosis, and nocardiosis can invade the chest wall, but usually do so in a single area rather than in multiple areas (38).

PET has an important role to play in the staging of MPM and should be used routinely. Utilizing fludeoxyglucose (FDG)-PET is the most commonly studied and used radionuclide imaging agent for mesothelioma and most thoracic malignancies and is a powerful diagnostic tool (39). MPM usually shows intense FDG uptake that matches the thickened pleura seen on CT or magnetic resonance imaging (MRI). It will also demonstrate involvement of the chest wall, fissure, and lymph node metastases. In a study by Bénard et al. in which 28 patients received FDG-PET followed by thoracoscopy, they demonstrated excellent overlap/matching between the burden of disease seen on PET and the burden of disease found on thoracoscopy, although in two cases FDG-PET significantly underestimated the burden of disease (one epitheloid, one biphasic) (39). Overall, they demonstrated that FDG-PET had a sensitivity of $92 \%$ and specificity of $75 \%$ for demonstration of malignant disease. They also performed semiquantitative analysis of 26 of their subjects and concluded that an SUV >2.0 was a reasonable cutoff to distinguish between malignant and benign pleural disease, with a sensitivity of $91 \%$ and a specificity of $100 \%$. Another study by Yildirim et al. demonstrated similar findings, evaluating 31 patients with pleural disease (17 MPM, 14 benign), and finding that FDG-PET had a sensitivity of $88 \%$ and specificity of $93 \%$ for malignant disease and was able to identify 13 out of 14 cases of benign disease (40). If correlated with patient specific information such as exposure history these metrics may improve further. Their mean SUV values were $6.5 \pm 3.4$ for MPM cases and $0.8 \pm 0.6$ for benign pleural diseases. Their analysis showed an SUV cutoff of 2.2 with a $94 \%$ sensitivity and $100 \%$ specificity. For post-surgery surveillance, FDG-PET may also be superior to contrast-enhanced CT for demonstrating recurrence and may have an impact on treatment algorithms (41).

Though not routinely utilized in surgical trails due to the requirement of an experienced radiologist, the role of MRI in diagnosis of MPM is primarily focused on determining resectability. MRI enables differentiation of tumor from normal adjacent tissue by using different pulse sequences and imaging in multiple planes but due to the motion of heart and breathing muscles, cardiac gating and respiratory compensation should be utilized $(42,43)$. MPM shows moderately increased signal on $\mathrm{T} 2$-weighted images that can be helpful for differentiation. Use of gadolinium-based IV contrast may also be helpful by increasing enhancement and contrast enhanced T1 fat-suppressed sequences are sensitive for detecting enhancement of the interlobar fissures and tumor invasion of surrounding structures $(36,44)$. MRI is superior to CT in terms of evaluating invasion of the diaphragmatic muscle (accuracy 0.82 vs. 0.55) and endothoracic fascia or single chest wall focus (accuracy 
0.69 vs. 0.46) (43). PET MRI may also play an increased role in the future to help more accurately stage patients particularly regarding their $\mathrm{T}$ stage, although widespread availability is likely the biggest limiting factor $(45,46)$.

Ultrasound has a limited role to play in the diagnosis of MPM but may be one of the first tests performed by a bedside physician when assessing a pleural effusion of unknown origin. Ultrasound can be used to assess volume and location of pleural fluid, nodular pleural thickening, irregular thickening in the fissures, or a localized mass (25). Pleural thickening greater than $1 \mathrm{~cm}$, nodular pleural thickening, pleural-based masses, and diaphragm nodules or thickening greater than $7 \mathrm{~mm}$ should all raise the suspicion for malignancy. In a series of 52 patients with suspected malignant pleural effusions, Qureshi et al. report a sensitivity for ultrasound of $73 \%$, specificity $100 \%$, PPV $100 \%$, NPV $73 \%$ for diagnosis of malignant disease (47). When ultrasound raises concern for MPM, cross sectional imaging and biopsy should be performed. Ultrasound can play a key role in image-guided thoracentesis for cytology of malignant effusion or core needle biopsy of suspicious pleural-based lesions.

\section{Conclusions}

MPM is a complex disease that causes significant morbidity and mortality and remains a challenging disease to diagnose and stage accurately, particularly as the different subtypes can behave differently. Nevertheless, accurate diagnosis of subtypes and diligent staging are important for making treatment decisions. Clinicians must also be mindful of how their diagnostic techniques may affect future treatment options. Higher resolution diagnostic imaging combined with functional imaging will likely play a greater role in future diagnosis and staging, and future updates to the staging system may incorporate some of this tumor thickness or volumetric data.

\section{Acknowledgments}

Funding: None.

\section{Footnote}

Provenance and Peer Review: This article was commissioned by the Guest Editors (Abbas E. Abbas and Stacey Su) for the series "Mesothelioma" published in AME Surgical fournal. The article has undergone external peer review.
Peer Review File: Available at https://asj.amegroups.com/ article/view/10.21037/asj-21-71/prf

Conflicts of Interest: All authors have completed the ICMJE uniform disclosure form (available at https://asj.amegroups. com/article/view/10.21037/asj-21-71/coif). The series "Mesothelioma" was commissioned by the editorial office without any funding or sponsorship. The authors have no other conflicts of interest to declare.

Ethical Statement: The authors are accountable for all aspects of the work in ensuring that questions related to the accuracy or integrity of any part of the work are appropriately investigated and resolved.

Open Access Statement: This is an Open Access article distributed in accordance with the Creative Commons Attribution-NonCommercial-NoDerivs 4.0 International License (CC BY-NC-ND 4.0), which permits the noncommercial replication and distribution of the article with the strict proviso that no changes or edits are made and the original work is properly cited (including links to both the formal publication through the relevant DOI and the license). See: https://creativecommons.org/licenses/by-nc-nd/4.0/.

\section{References}

1. Pass H, Giroux D, Kennedy C, et al. The IASLC Mesothelioma Staging Project: Improving Staging of a Rare Disease Through International Participation. J Thorac Oncol 2016;11:2082-8.

2. Richards WG, Godleski JJ, Yeap BY, et al. Proposed adjustments to pathologic staging of epithelial malignant pleural mesothelioma based on analysis of 354 cases. Cancer 2010;116:1510-7.

3. Sugarbaker DJ, Flores RM, Jaklitsch MT, et al. Resection margins, extrapleural nodal status, and cell type determine postoperative long-term survival in trimodality therapy of malignant pleural mesothelioma: results in 183 patients. J Thorac Cardiovasc Surg 1999;117:54-63; discussion 63-5.

4. Cameron RB. Staging in the era of international databases: documented improvements with remaining challenges. J Thorac Dis 2018;10:682-7.

5. Rusch VW. A proposed new international TNM staging system for malignant pleural mesothelioma. From the International Mesothelioma Interest Group. Chest 1995;108:1122-8.

6. Berzenji L, Van Schil PE, Carp L. The eighth TNM 
classification for malignant pleural mesothelioma. Transl Lung Cancer Res 2018;7:543-9.

7. Kindler HL. Robust data: the essential foundation of a revised staging system for pleural mesothelioma. J Thorac Oncol 2012;7:1623-4.

8. Nowak AK, Chansky K, Rice DC, et al. The IASLC Mesothelioma Staging Project: Proposals for Revisions of the T Descriptors in the Forthcoming Eighth Edition of the TNM Classification for Pleural Mesothelioma. J Thorac Oncol 2016;11:2089-99.

9. Tertemiz KC, Ozgen Alpaydin A, Gurel D, et al. Multiple distant metastases in a case of malignant pleural mesothelioma. Respir Med Case Rep 2014;13:16-8.

10. Beasley MB, Galateau-Salle F, Dacic S. Pleural mesothelioma classification update. Virchows Arch 2021;478:59-72.

11. Kindler HL, Ismaila N, Armato SG 3rd, et al. Treatment of Malignant Pleural Mesothelioma: American Society of Clinical Oncology Clinical Practice Guideline. J Clin Oncol 2018;36:1343-73.

12. Hwang HC, Sheffield BS, Rodriguez S, et al. Utility of BAP1 Immunohistochemistry and p16 (CDKN2A) FISH in the Diagnosis of Malignant Mesothelioma in Effusion Cytology Specimens. Am J Surg Pathol 2016;40:120-6.

13. Agarwal PP, Seely JM, Matzinger FR, et al. Pleural mesothelioma: sensitivity and incidence of needle track seeding after image-guided biopsy versus surgical biopsy. Radiology 2006;241:589-94.

14. Heilo A, Stenwig AE, Solheim OP. Malignant pleural mesothelioma: US-guided histologic core-needle biopsy. Radiology 1999;211:657-9.

15. Welch BT, Eiken PW, Atwell TD, et al. A SingleInstitution Experience in Percutaneous Image-Guided Biopsy of Malignant Pleural Mesothelioma. Cardiovasc Intervent Radiol 2017;40:860-3.

16. Adams RF, Gray W, Davies RJ, et al. Percutaneous imageguided cutting needle biopsy of the pleura in the diagnosis of malignant mesothelioma. Chest 2001;120:1798-802.

17. Wald O, Groth SS, Burt BM, et al. Role of thoracoscopy, mediastinoscopy and laparoscopy in the diagnosis and staging of malignant pleural mesothelioma. J Vis Surg 2016;2:129.

18. Scherpereel A, Opitz I, Berghmans T, et al. ERS/ESTS/ EACTS/ESTRO guidelines for the management of malignant pleural mesothelioma. Eur Respir J

19. Rahman NM, Ali NJ, Brown G, et al. Local anaesthetic thoracoscopy: British Thoracic Society Pleural Disease Guideline 2010. Thorax 2010;65 Suppl 2:ii54-60.
20. Haridas N, K P S, T P R, et al. Medical Thoracoscopy vs Closed Pleural Biopsy in Pleural Effusions: A Randomized Controlled Study. J Clin Diagn Res 2014;8:MC01-4.

21. Yanagawa J, Rusch V. Surgical management of malignant pleural mesothelioma. Thorac Surg Clin 2013;23:73-87, vi.

22. Bölükbas S, Eberlein $M$, Kudelin N, et al. Factors predicting poor survival after lung-sparing radical pleurectomy of IMIG stage III malignant pleural mesothelioma. Eur J Cardiothorac Surg 2013;44:119-23.

23. Rice DC, Steliga MA, Stewart J, et al. Endoscopic ultrasound-guided fine needle aspiration for staging of malignant pleural mesothelioma. Ann Thorac Surg 2009;88:862-8; discussion 868-9.

24. Czarnecka-Kujawa K, de Perrot M, Keshavjee S, et al. Endobronchial ultrasound-guided transbronchial needle aspiration mediastinal lymph node staging in malignant pleural mesothelioma. J Thorac Dis 2019;11:602-12.

25. Bibby AC, Tsim S, Kanellakis N, et al. Malignant pleural mesothelioma: an update on investigation, diagnosis and treatment. Eur Respir Rev 2016;25:472-86.

26. Hollevoet K, Reitsma JB, Creaney J, et al. Serum mesothelin for diagnosing malignant pleural mesothelioma: an individual patient data meta-analysis. J Clin Oncol 2012;30:1541-9.

27. Cui A, Jin XG, Zhai K, et al. Diagnostic values of soluble mesothelin-related peptides for malignant pleural mesothelioma: updated meta-analysis. BMJ Open 2014;4:e04145.

28. Park EK, Sandrini A, Yates DH, et al. Soluble mesothelinrelated protein in an asbestos-exposed population: the dust diseases board cohort study. Am J Respir Crit Care Med 2008;178:832-7.

29. Felten MK, Khatab K, Knoll L, et al. Changes of mesothelin and osteopontin levels over time in formerly asbestos-exposed power industry workers. Int Arch Occup Environ Health 2014;87:195-204.

30. Ahmadzada T, Reid G, Kao S. Biomarkers in malignant pleural mesothelioma: current status and future directions. J Thorac Dis 2018;10:S1003-7.

31. Pass HI, Alimi M, Carbone $M$, et al. Mesothelioma Biomarkers: A Review Highlighting Contributions from the Early Detection Research Network. Cancer Epidemiol Biomarkers Prev 2020;29:2524-40.

32. Gill RR, Yeap BY, Bueno R, et al. Quantitative Clinical Staging for Patients With Malignant Pleural Mesothelioma. J Natl Cancer Inst 2018;110:258-64.

33. Gill RR, Naidich DP, Mitchell A, et al. North American Multicenter Volumetric CT Study for Clinical Staging of 
Malignant Pleural Mesothelioma: Feasibility and Logistics of Setting Up a Quantitative Imaging Study. J Thorac Oncol 2016;11:1335-44.

34. Kawashima A, Libshitz HI. Malignant pleural mesothelioma: CT manifestations in 50 cases. AJR Am J Roentgenol 1990;155:965-9.

35. Sahin AA, Cöplü L, Selçuk ZT, et al. Malignant pleural mesothelioma caused by environmental exposure to asbestos or erionite in rural Turkey: CT findings in 84 patients. AJR Am J Roentgenol 1993;161:533-7.

36. Yamamuro M, Gerbaudo VH, Gill RR, et al. Morphologic and functional imaging of malignant pleural mesothelioma. Eur J Radiol 2007;64:356-66.

37. Leung AN, Müller NL, Miller RR. CT in differential diagnosis of diffuse pleural disease. AJR Am J Roentgenol 1990;154:487-92.

38. Bonomo L, Feragalli B, Sacco R, et al. Malignant pleural disease. Eur J Radiol 2000;34:98-118.

39. Bénard F, Sterman D, Smith RJ, et al. Metabolic imaging of malignant pleural mesothelioma with fluorodeoxyglucose positron emission tomography. Chest 1998;114:713-22.

40. Yildirim H, Metintas M, Entok E, et al. Clinical value of fluorodeoxyglucose-positron emission tomography/ computed tomography in differentiation of malignant mesothelioma from asbestos-related benign pleural disease: an observational pilot study. J Thorac Oncol 2009;4:1480-4.

41. Kitajima K, Hashimoto M, Katsuura T, et al. Clinical

doi: $10.21037 /$ asj-21-71

Cite this article as: Jordan S, Nitsche L, Yendamuri S. Review of staging and diagnosis of malignant pleural mesothelioma. AME Surg J 2022;2:28. utility of FDG-PET/CT for post-surgery surveillance of malignant pleural mesothelioma - Comparison with contrast-enhanced CT. Oncotarget 2019;10:6816-28.

42. Truong MT, Viswanathan C, Godoy MB, et al. Malignant pleural mesothelioma: role of CT, MRI, and PET/CT in staging evaluation and treatment considerations. Semin Roentgenol 2013;48:323-34.

43. Heelan RT, Rusch VW, Begg CB, et al. Staging of malignant pleural mesothelioma: comparison of CT and MR imaging. AJR Am J Roentgenol 1999;172:1039-47.

44. Knuuttila A, Kivisaari L, Kivisaari A, et al. Evaluation of pleural disease using MR and CT. With special reference to malignant pleural mesothelioma. Acta Radiol 2001;42:502-7.

45. Martini K, Meier A, Opitz I, et al. Diagnostic accuracy of sequential co-registered $\mathrm{PET}+\mathrm{MR}$ in comparison to PET/CT in local thoracic staging of malignant pleural mesothelioma. Lung Cancer 2016;94:40-5.

46. Murphy DJ, Mak SM, Mallia A, et al. Loco-regional staging of malignant pleural mesothelioma by integrated 18F-FDG PET/MRI. Eur J Radiol 2019;115:46-52.

47. Qureshi NR, Rahman NM, Gleeson FV. Thoracic ultrasound in the diagnosis of malignant pleural effusion. Thorax 2009;64:139-43. 